\title{
Prospects for the Development of Digital Healthcare Sector through Patent Analysis
}

\author{
Wan Sup Um¹, Young Hun Kim², Jun Cheul Park ${ }^{1}$ \\ ${ }^{1}$ Department of Industrial \& Management Engineering, Gangneung-Wonju National University, Wonju, Republic of Korea \\ ${ }^{2}$ Gangwon Economic Promotion Agency, Wonju, Republic of Korea \\ Email: eomeom@gwnu.ac.kr, gns0001@gwep.or.kr, jcking@gwnu.ac.kr
}

How to cite this paper: Um, W. S., Kim, Y. H., \& Park, J. C. (2020). Prospects for the Development of Digital Healthcare Sector through Patent Analysis. iBusiness, $12,160-173$.

https://doi.org/10.4236/ib.2020.124012

Received: November 10, 2020

Accepted: December 20, 2020

Published: December 23, 2020

Copyright $\odot 2020$ by author(s) and Scientific Research Publishing Inc. This work is licensed under the Creative Commons Attribution International License (CC BY 4.0).

http://creativecommons.org/licenses/by/4.0/ (c) (i) Open Access

\begin{abstract}
In recent years, the healthcare information system based on big data has been growing rapidly and is being adapted to medical information to derive important health trends and support timely preventive care. This study analyzes patents to grasp the latest technological trends and core competencies in the digital healthcare field in Korea, by identifying which fields have numerous patent applications, and which fields have few patent applications. The technology classification system for patent analysis is divided into health information measurement technology (A), healthcare platform technology (B), and healthcare teleservice technology $(\mathrm{C})$ for digital healthcare. Korea is confirmed to have high patented technology in the field of biometric measurement technology and health information building, sharing, and utilization technology. In the field of life information pattern measurement technology, the patent activity rate is high, while the market retention rate is very low. As a result of OS Matrix analysis, in the field of Korean healthcare, technology development using wearable devices or toilet seats for identification, diagnosis, and treatment of users, and patient pattern analysis using big data were found to be areas with relatively low concentration of R\&D or patent applications. And it seems that there is a need for technology development and patent application in these fields in the future.
\end{abstract}

\section{Keywords}

Digital Healthcare, Patent Information, Patent Index, OS Matrix

\section{Introduction}

A majority of people do not know or care much about their health. Even mild diseases, such as colds, can lead to serious diseases if neglected by indifference and ignorance, and diseases such as viruses or AIDS can be a bigger problem. 
Therefore, it is best to prevent this disease in advance. In fact, Korea's health care system is shifting from treatment-oriented to preventive care and health management (Jung et al., 2015).

In Korea, the national medical insurance system was introduced before the 1970s, and hospital computerization was carried out substantially. In the 1980s, medical information was rapidly digitalized. In the 1990s, primary medical institutions at the clinic level began to introduce the Electronic Medical Record (EMR) system, and major domestic university hospitals began to introduce EMR in earnest from the mid-2000s. Thus, the tremendous accumulation of clinical data in hospitals has laid the foundation for digital healthcare. However, in the case of Korea, there are a number of restrictions on the creation or use of medical big data due to various regulatory policies such as excessive personal information regulation, public information regulation, specific cloud usage regulation, and structural problems for medical services (Kim, 2019; Shim \& Shim, 2019; Kim et al., 2017).

Health care includes not only the treatment of diseases, but also prevention and health management, and digital health care is defined as an industrial area that manages personal health and diseases by fusion of the healthcare industry and ICT. Healthcare data are divided into three categories: exogenous data, genomic data, and medical data. Of the three categories, exogenous data are composed of socioeconomic and environmental factors and account for $99.4 \%$ of the total data. Here, big data technology that creates meaningful information through the development of information communication and data accumulation technology is being actively used in the medical field.

The National Health Insurance Service operates a national health alert system and provides alarm services for major diseases such as colds, eye diseases, food poisoning, asthma, and dermatitis. Based on treatment data by period and the frequency of keywords on SNS, the region and age are determined. It operates a system that predicts diseases while providing precautions in advance by categorizing them into four levels: interest, caution, warning, and risk. In addition, due to the advancement of the medical information system and the rapid development of IT, enormous medical big data information is accumulating, and has great significance in solving the problem of aging population and increasing medical costs. The importance of this field cannot be understated and can greatly influence the healthcare service system (Jung et al., 2018).

Meanwhile, it is now possible to easily self-diagnose and monitor personal health through various healthcare apps on smartphones. For example, it is possible to check from simple health conditions to complex conditions such as calorie consumption, eating habits, blood pressure, and sleep measurement. This has led to a huge accumulation of personal medical data.

This study aims to provide reliable and effective management information where medical services are needed by linking and analyzing patent information with healthcare big data and identify technological trends through a patent analysis on digital healthcare. First, by researching and analyzing the patents in 
the healthcare field of major countries, the technology trends are confirmed, and secondly, the technical competitiveness of Korean companies in the healthcare field is analyzed through technology megatrend and technology competitiveness analysis (major patent index, etc.), and finally, by using object and solution matrix analysis, we are attempting to determine the field where patents are not active yet or where technology development is required to ascertain the promising technical fields of digital healthcare in Korea.

\section{Literature Review}

\subsection{Big Data}

Big data are defined as "a technology for collecting and analyzing large amounts of data that is difficult to handle with existing information management and analysis systems". Gartner's " $3 \mathrm{Vs"}$ model is used to define the properties volume, velocity, and variety of these big data (Laney, 2001). Big data analysis is to extract and visualize meaningful information from numerous heterogeneous data. In addition to general laboratory data, there are various types of data, numbers and text images accumulated in the workplace of a company (Kim \& Kim, 2019).

In the field of big data, many domestic and foreign companies have recently participated in R\&D, and some are showing some performance. Currently, in foreign countries, Google, Amazon, IBM, EMC, Oracle, SAP, etc. are making efforts to enter the big data business and secure technology by taking advantage of their respective strengths, and intend to introduce a big data platform. In Korea, search engine development companies such as Wisenut, Conan Technology, Diquest, and Saltlux are going to enter the big data field, but are still showing products in the early stages, and database companies such as Altibase, Tmaxdata, and Datastreams, and Portal companies such as Naver, Kakao are developing big data solutions. Among the many types of big data, medical big data are important because it has high growth potential. In addition, there is a background of "population aging" in Korea. In order for the healthcare industry to grow, medical big data and cloud-based medical big data can be combined to enable remote medical treatment and provide powerful artificial support medical services. However, there is a lack of medical big data due to restrictions on personal information and specific cloud use in Korea. Looking at cloud-based traffic, the US is $86 \%$, but Korea is only $1.8 \%$ (Jung \& Lee, 2017).

\subsection{Healthcare}

In general, healthcare data contains sensitive information, typically personal information. The problem of personal information protection must be resolved, and in order to analyze healthcare data, the approval of the Research Ethics Committee must be obtained first. The Research Ethics Review Committee does not review the scientific method of the research, but approves the research by reviewing whether the research is harmful to the patients. In addition to these 
problems, most of the healthcare data are used to improve the quality of life of individuals. Recently, with the development of machine learning techniques, big data utilization methods using artificial intelligence have been widely used.

As of 2013, the global healthcare market amounted to USD 6.15 trillion and is expected to grow at an annual average of $12 \%-16 \%$. The growth of the healthcare industry has a background of "population aging," and along with Japan, Korea's population aging is progressing at a rapid pace. The number of people over 65 in Korea is expected to increase from $13.1 \%$ in 2015 to $24.3 \%$ in 2030 and $40.1 \%$ in 2060 (Korea Statistics, 2015). According to the National Health Insurance Service, Korea entered an aging society in 2016, and as of the medical expenses used by the elderly over 65 years old, $14 \%$ of the population, amounted to 25.187 billion won in 2016, accounting for $40 \%$ of the total medical expenses (Jung \& Lee, 2017). The research on healthcare big data is largely divided into two directions. The first is the use of healthcare big data and the protection of personal information. Two such cases of information leakage occurred by an American insurance company Anthem in 2014 and the Korea Pharmacy Information Service in 2010 As seen in the case where patient information was provided to IMS, a medical statistics company and paid for, it was about the development of various methods or personal information protection processes for data de-identification prior to data use for personal information protection (Kim et al., 2017; Kim, 2019).

And the second is divided into research that develops industrial development or people's health care service platform using artificial intelligence techniques with big data (Kim et al., 2014; Nkenyereye \& Jang, 2015; Lim et al., 2017; Kim, 2017; Jung et al., 2015).

Health care data are generated during the process of treating patients in hospitals and is stored by the hospital. When looking at the types of data, it has a wide variety of forms that are text such as medical records, images such as CT and MRI, videos such as ultrasound and endoscopy images, and codes, such as diagnosis and drug names. In addition to data generated by hospitals, it is composed of various data, such as genome, environmental information, life activity information, climate environment data, food and exercise, and mental health data. Among the healthcare data that individuals generate throughout their lifetime, the size of medical information held by hospitals is about $0.4 \mathrm{~TB}$, while genomic data is $6 \mathrm{~TB}$, and environmental and daily life data reaches $1100 \mathrm{~TB}$.

The effects of these data on health are $10 \%$ of medical information and $30 \%$ of genome information, and the remaining data account for $60 \%$. In Korea, various healthcare data are open to the public, and representative data are summarized in Table 1 (Shin, 2017).

\section{Patent Analysis}

\subsection{Analysis Target}

In this study, digital healthcare patents were analyzed using the Wisdomain DB 
Table 1. Public healthcare data in Korea.

\begin{tabular}{lll}
\hline \multicolumn{1}{c}{ Agency } & \multicolumn{1}{c}{ Data name } & URL \\
\hline & Million Sample Cohort DB & $\underline{\text { https://nhiss.nhis.or.kr/bd/ab/bdaba002cv.do }}$ \\
& Health Examination Cohort DB & $\underline{\text { https://nhiss.nhis.or.kr/bd/ab/bdaba006cv.do }}$ \\
National Health Insurance Corporation & Senior Cohort DB & $\underline{\text { https://nhiss.nhis.or.kr/bd/ab/bdaba008cv.do }}$ \\
& Working Women Cohort DB & $\underline{\text { https://nhiss.nhis.or.kr/bd/ab/bdaba009cv.do }}$ \\
& Infant and Infant Screening Cohort DB & $\underline{\text { https://nhiss.nhis.or.kr/bd/ab/bdaba010cv.do }}$ \\
Health Insurance Review and Assessment Service & Health and Medical Big Data Open System $\underline{\underline{\text { http://opendata.hira.or.kr/ }}}$ \\
The Korea Disease Control and Prevention Agency & National Health and Nutrition Survey & $\underline{\text { http://www.kdca.go.kr/ }}$ \\
National Cancer Center & National Cancer Registration Data & $\underline{\text { http://www.ncc.re.kr/main.ncc?uri=manage02_4 }}$ \\
Korea Institute for Health and Social Affairs & Korea Medical Panel & $\underline{\text { http://www.khp.re.kr/ }}$ \\
\hline
\end{tabular}

(https://www.wisdomain.com/). In Korea, with the introduction of the national health insurance system, medical information has been rapidly digitized, and major university hospitals have introduced electronic medical record systems since the 2000s. We analyzed the patent applications and registered Korean, US, Japanese, Chinese and European patents from January 2001 to April 2020.

In the preceding research, a healthcare patent analysis was conducted using Wisdomain DB for patent applications and registered patents in Korea, US, Japan, China, and Europe from 1975 to 2018. Technology competitiveness was analyzed, and texture mining was used for technology trend analysis (Kim et al., 2018).

The technology classification system used for health care patent analysis in this study has a health information measurement technology (A), a healthcare platform technology (B), and healthcare teleservice technology (C). These technologies were further subdivided and used for patent search. Health information measurement technology (A) was subdivided into biometric measurement technology (A1) and life information pattern measurement technology (A2), and healthcare platform technology (B) was subdivided into health information building, sharing and utilization technology (B1), health information big data analytics technology (B2) and mobile healthcare service platform technology (B3). Healthcare teleservice technology (C) was subdivided into remote monitoring technology (C1), customized remote care technology (C2), and customized remote treatment technology (C3). The technology tree related to digital healthcare is summarized in Table 2 (Kim et al., 2018).

\subsection{Analysis Items}

\subsubsection{Number of Patents by Technology}

Companies that want to analyze country-specific patents by technology field to grasp the technological level of each country and to pursue commercialization in a specific country, if they intend to do business by applying a patent in that country, grasp the number of patents by technology and consider the technology 
Table 2. Technology tree related to digital healthcare.

\begin{tabular}{cl}
\hline Technical Classification & \multicolumn{1}{c}{ Detailed Technical Classification } \\
\hline $\begin{array}{c}\text { Health Information } \\
\text { (A) }\end{array}$ & A1. Biometric measurement technology \\
A2. Life information pattern measurement technology \\
Healthcare Platform & B1. Health information building, sharing, and utilization technology \\
Technology & B2. Health information big data analytics technology \\
(B) & B3. Mobile healthcare service platform technology \\
Healthcare Teleservice & C1. Remote monitoring technology \\
Technology & C2. Customized remote care technology \\
(C) & C3. Customized remote treatment technology \\
\hline
\end{tabular}

(Kim et al., 2018).

trend by marketability.

\subsubsection{Main Applicant}

There are numerous methods to ascertain Korean patented technology, but it is also meaningful to first identify the applicants and countries that are making investments, research, and commercialization in the digital healthcare field.

\subsubsection{Analysis Using Patent Index}

The patent index is widely used as a measurement tool for commercialization activities as a method of measuring output versus input for science and technology. Based on the registered patent data, the development of science, technology and industry can be promoted by using the patent analysis index. The patent index is generally divided into indexes for analysis of the main technology field, citation related indexes, and other indexes.

Indices for the analysis of key technical fields include the Patent Activity Index (AI), the Patent Family Size (PFS), and the Cites Per Patent (CPP) and Patent Impact Index (PII), and other indices include Technology Strength (TS) and Science Linkage (SL). In our study, five indices such as AI, PFS, CPP, PII, and TS were analyzed.

1) Patent Activity Index (AI)

It is an index to find out what field a specific company (country) is specialized in. It is an index indicating the patent concentration of a specific technology among all patents of a company. If AI is 1 or more, it means that the company's patent activity is higher than the average in the relevant technology field, and a technology or company with AI 3 or more is called a high specialized technology or a high specialized company.

Total number of patents of specific technology by specific applicant

$$
\mathrm{AI}=\frac{\text { Total number of patents of specific technology }}{\frac{\text { Total number of patents by specific applicant }}{\text { Total number of patents }}}
$$

2) Market Security Index (PFS: Patent Family Size) 
Patents filed in multiple countries for one invention are called family patents. In many cases, patents are filed overseas only when there is a commercial interest or technical competition in the country, so the greater the number of family patents the greater the market securing power, and this is used as an indicator of market securing power.

$$
\text { PFS }=\frac{\text { Average number of patent families of the applicant }}{\text { Total average number of patent families }}
$$

3) Cites Per Patent (CPP)

It is the average value of the number of times that the patents of a specific patentee are cited by the patents that are subsequently registered, and the larger this value means that they have major or original patents, and the patent holders with many cited patents have an advantage in competition.

$$
\mathrm{CPP}=\frac{\text { Citation Count }}{\text { Number of patents }}
$$

4) Influence Index (PII: Patent Impact Index)

Influence index reflects past technical activity based on a point in time, and measures the quality of technology owned by a specific applicant (patent holder). If PII is 1 , it indicates the average citation frequency, and if it is 2 , it indicates that it is cited with twice the frequency of the average.

$$
\begin{aligned}
\text { PII } & =\frac{\text { Citation ratio of specific applicants in specific technical fields }}{\text { Total Citation Rate }} \\
& =\frac{\text { Number of citations in specific technical fields/Number of patents in specific technical fields }}{\text { Total number of citations/Total number of patents }}
\end{aligned}
$$

\section{5) Technology Strength (TS)}

The multiplication of the influence index by the number of patents is called the technology strength index, and the higher the technology strength index, the higher the technology level of the relevant country or research subject.

$$
\text { TS }=\text { Number of patents } \times \text { Influence Index }(\mathrm{PII})
$$

\subsubsection{Technology Trend Analysis}

In order to analyze technology trends using digital healthcare related patents, the results derived using text mining can be grasped through word cloud. Word cloud is a tool that draws out and visualizes key words from text or data, and it is a technique that allows anyone to easily identify keywords because it is visualized by increasing the size of words in proportion to the frequency of occurrence of words. In our study, keywords were analyzed using the word cloud provided by Wisdomain for the specifications of patents applied or registered from January 2001 to April 2020.

First, let's analyze the word cloud for health information measurement technology (A), healthcare management platform technology (B), and remote service technology $(C)$ for healthcare. 
Figure 1 shows the word cloud of health information measurement technology (A). Keywords of that are health, data, life, service, monitoring and patient. The keywords related to digital measurement devices are appeared the most. We use the technology related to measuring our health while living our daily lives without visiting a hospital using a wireless device. Technology that secures desired personal medical information by detecting and finding it is seen as a major technology trend.

In addition, in the healthcare platform technology (B), keywords such as health, data, service, server, and individual appear the most (See Figure 2). Therefore, the technology of building a mobile-based healthcare service platform that can receive services anywhere while always monitoring health information seems to be a major technology trend. However, in order to make this possible, technologies related to health information sharing and health information big data must also be implemented.

Figure 3 shows the word cloud of healthcare teleservice technology (C). In the healthcare teleservice technology (C), keywords such as health, service, platform, communication, network, monitoring, care, cloud, and data appeared most frequently. As technologies in the field of healthcare big data are emerging, the

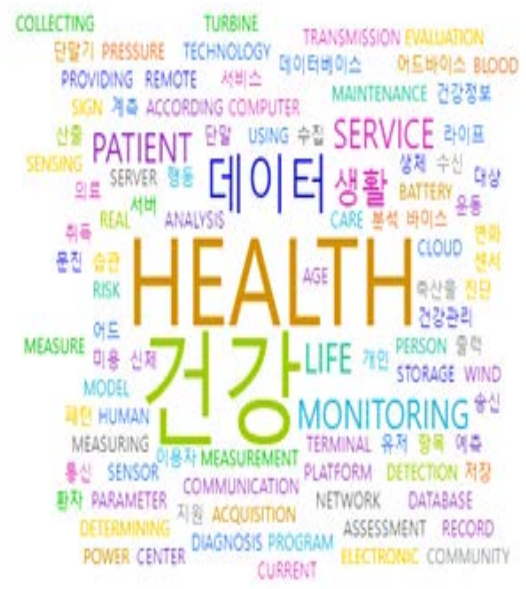

Figure 1. Word cloud of health information measurement technology (A).

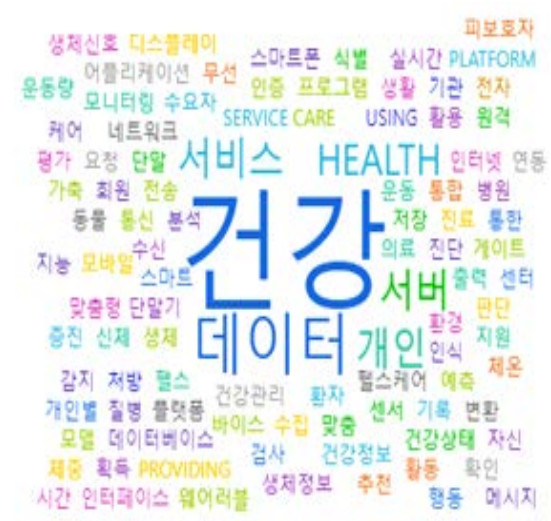

Figure 2. Word cloud of healthcare platform technology (B). 


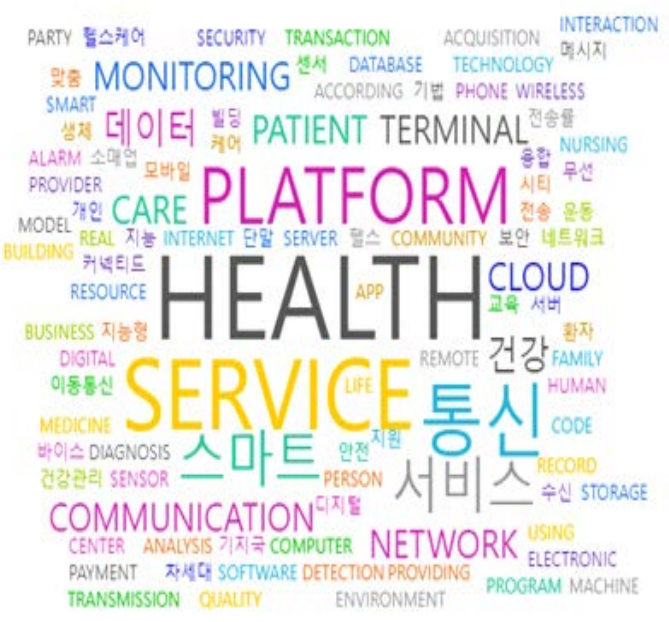

Figure 3. Word cloud of healthcare teleservice technology (C).

development of service products using smart wireless devices seems to be a major technology trend for platforms that can perform healthcare services along with communication infrastructure.

\section{Analysis Result}

\subsection{No. of Patents by Detailed Technology}

The total number of patents to be analyzed in this study was 3786, of which 1337 patent applications were filed in Korea, the highest (35.3\%), followed by 935 (24.7\%) in the United States, 746 (19.7\%) in the Japan, 715 cases (18.9\%) in China, and 53 (1.4\%) in Europe (See Table 3). Looking at patents by detailed technology, patents for biometric measurement technology (A1) were searched the highest with 1243 (32.8\%), secondly, customized teleservice technology (C3) was $688(18.2 \%)$, followed by Mobile healthcare service platform technology (B3), lifestyle information pattern measurement technology (A2), and health information building, sharing and utilization technology (B1), and health information measurement technology (A) accounted for $44.6 \%$ with a total of 1,687 cases.

\subsection{Major Patent Applicants}

Table 4 shows 10 major patent applicants. In terms of patent applicants, Samsung Electronics Co., Ltd. of Korea took the first place with 101 cases, including the headquarters in Korea and branches in Japan and China, and Matsushida Electric Industries Co., Ltd. of Japan ranked second with 42 cases, and the Electronics and Telecommunications Research Institute ranked 3rd with 38 cases. Among the top ten applicants, there are 5 Korean nationals, 3 Japanese companies, and 1 US and China. A few years ago, there was a research report that Japanese companies applied for many patents, but it was confirmed that Korean companies are making a lot of investment and effort now. However, in the case of Korea, there is a problem that the contribution of Samsung Electronics Co., 
Table 3. Patents related to digital healthcare.

\begin{tabular}{|c|c|c|c|c|c|c|c|}
\hline Technical Classification & Detailed Technical Classification & $\mathrm{CN}$ & EP & JP & $\mathrm{KR}$ & US & total \\
\hline \multirow{2}{*}{$\begin{array}{c}\text { Health Information } \\
\text { Measurement Technology } \\
\text { (A) }\end{array}$} & A1. Biometric measurement technology & 80 & 14 & 368 & 464 & 317 & 1243 \\
\hline & A2. Life information pattern measurement technology & 165 & 9 & 94 & 62 & 114 & 444 \\
\hline Healthcare Platform & B1. Health information building, sharing, and utilization technology & 12 & 0 & 19 & 325 & 12 & 368 \\
\hline \multirow{2}{*}{$\begin{array}{c}\text { Technology } \\
\text { (B) }\end{array}$} & B2. Health information big data analytics technology & 28 & 1 & 1 & 73 & 8 & 111 \\
\hline & B3. Mobile healthcare service platform technology & 225 & 0 & 4 & 159 & 72 & 460 \\
\hline Healthcare Teleservice & C1. Remote monitoring technology & 87 & 4 & 21 & 24 & 110 & 246 \\
\hline \multirow{3}{*}{$\begin{array}{c}\text { Technology } \\
\text { (C) }\end{array}$} & C2. Customized remote care technology & 27 & 2 & 52 & 92 & 53 & 226 \\
\hline & C3. Customized remote treatment technology & 91 & 23 & 187 & 138 & 249 & 688 \\
\hline & Total & 715 & 53 & 746 & 1337 & 935 & 3786 \\
\hline
\end{tabular}

Table 4. Major applicants.

\begin{tabular}{cc}
\hline Patent Applicant & Number of patent applications \\
\hline Samsung Electronics Co., Ltd. & 101 \\
Matsushida Electric Industry Co., Ltd. & 42 \\
Electronics and Telecommunications Research Institute & 38 \\
Health Hero Network, Inc. & 24 \\
SK Telecom Co., Ltd. & 24 \\
LilYung & 17 \\
LG Electronics Co., Ltd. & 16 \\
Japan Telegraph Telephone Co., Ltd. & 15 \\
KT Co., Ltd. & 15 \\
Tanita Co., Ltd. & 15 \\
\hline
\end{tabular}

Ltd. is too large, and future technological development efforts are needed to explore new areas with creativity.

\subsection{Patent index}

Table 5 shows the results of analyzing technological competitiveness by detailed technology field. The patent activity index is an index representing the patent concentration of a specific technology among all patents of a specific company or institution. As a result of analyzing the patent concentration by detailed technology field, the health information sharing and utilization technology field (B1) is the highest at 2.50. In addition, the field of remote monitoring technology $(\mathrm{C} 1)$ was the lowest at 0.28 . In the case of Korea, the activity index is slightly higher than the global average. The market acquisition index shows marketability through patents, with Japan the highest at 35.59, followed by the US at 24.29, followed by Europe at 23.22. Korea is 18.86, slightly higher than China's 15.18, but it can be seen that the marketability is lower than that of Japan, the United 
Table 5. Patent index.

\begin{tabular}{cccccccccccc}
\hline \multirow{2}{*}{$\begin{array}{c}\text { Detailed } \\
\text { Technical } \\
\text { Classification }\end{array}$} & \multicolumn{1}{c}{ Patent Activity } & \multicolumn{2}{c}{$\begin{array}{c}\text { Market } \\
\text { Acquisition }\end{array}$} & \multicolumn{2}{c}{$\begin{array}{c}\text { Cites Per } \\
\text { Patent }\end{array}$} & \multicolumn{2}{c}{ Patent Impact } & \multicolumn{2}{c}{$\begin{array}{c}\text { Technology } \\
\text { Strength }\end{array}$} \\
\cline { 2 - 11 } & Total & Korea & Total & Korea & Total & Korea & Total & Korea & Total & Korea \\
\hline A1 & 0.95 & 1.06 & 3.39 & 2.89 & 8.70 & 1.35 & 0.32 & 1.07 & 135.04 & 498.43 \\
A2 & 1.19 & 0.40 & 2.65 & 2.50 & 7.12 & 0.90 & 1.00 & 0.95 & 106.96 & 58.87 \\
B1 & 0.61 & 2.50 & 1.90 & 2.60 & 23.24 & 0.89 & 0.96 & 0.89 & 68.17 & 287.81 \\
B2 & 0.84 & 1.86 & 2.54 & 2.77 & 3.71 & 0.59 & 11.04 & 1.00 & 27.16 & 73.26 \\
B3 & 0.85 & 0.98 & 2.75 & 2.95 & 5.70 & 0.76 & 0.75 & 0.81 & 89.38 & 129.51 \\
C1 & 1.11 & 0.28 & 3.17 & 1.58 & 18.94 & 1.07 & 1.57 & 0.87 & 107.65 & 20.93 \\
C2 & 0.91 & 1.15 & 3.23 & 1.95 & 15.17 & 1.16 & 0.41 & 0.95 & 27.65 & 87.65 \\
C3 & 1.30 & 0.57 & 3.80 & 1.62 & 11.32 & 1.86 & 1.12 & 1.31 & 195.19 & 180.53 \\
Average & 0.97 & 1.10 & 2.93 & 2.36 & 11.74 & 1.07 & 2.15 & 0.98 & 94.65 & 167.12 \\
\hline
\end{tabular}

States, and Europe. In Japan, it was the highest in the mobile healthcare service platform technology field (B3) at 7.50, and in Korea, it was also the highest in the mobile healthcare service platform technology field (B3), but the index was 2.95 .

In terms of market acquisition index, the global average was 2.93, but in Korea it was low at 2.36. Korea's market acquisition index was generally low, and the field of remote monitoring technology ( $\mathrm{C} 1)$ was the lowest at 1.58. The higher the value of the citation index, the more important patents and original patents are held. The global average is 11.74, but in the case of Korea, the citation index is very low at 1.07, indicating that it is necessary to secure important original patents. In particular, the field of health information big data analysis technology (B2) is the lowest at 0.59 , indicating that investment in this field is necessary. Considering jointly with the market acquisition index, the quality level of US patents is high, but the market acquisition index is relatively low. In other words, market acquisition index is not as high as the quality level of US patents. In the case of Japan, there are not many original patents, but market acquisition index is the highest.

The influence index is an index that measures the qualitative level of technology owned by a specific applicant. In Korea, the index is generally low, and in particular, the index of the mobile healthcare service platform technology (B3) field was the lowest at 0.81 . In Europe, the technology in the field of health information big data analysis technology (B2) was the highest. In the case of the technology strength index, it was found that Korea's technological strength was relatively high, and the biometric measurement technology (A1) field was 498.43 , and the influence index was low at 1.07 , but the number of patents was very high. The remote monitoring technology (C1) field, which is also the weakest in the patent activity index and market acquisition index, shows a lower value compared to other countries in the technology strength index, so it seems that continuous interest and investment in this field is needed. 


\subsection{Technical Competitiveness Analysis}

\subsubsection{Reverse Technology Tree Analysis Centered on Core Patents}

Regardless of the existing technology classification system, in order to analyze the patent technology as a solution task and solution, and to create a reverse technology tree based on this, and to use it in future product research and development, first classify the problem to be solved for the core patent into keywords group. When Korean digital healthcare related patents are classified by purpose, they can be classified as monitoring, diagnosis, treatment, user identification, and a combination of several technologies. In addition, looking at the solutions used to achieve this purpose, it can be divided into IoT, mobile phones, networks, health activity information, big data, and wearable devices.

\subsubsection{Object and Solution Matrix}

Table 6 is the OS Matrix in Korean patents related digital healthcare. The main task areas in the OS Matrix are monitoring (489 cases), convergence (technology including two or more of monitoring, diagnosis, and treatment) (231 cases), diagnosis ( 89 cases), user identification ( 73 cases), treatment (45 cases). It is analyzed that the patents were distributed. The main solution areas are IoT (273 cases), mobile phones (220 cases), networks (187 cases), health activity information (99 cases), big data (78 cases), and wearable devices ( 70 cases).

As a result of OS Matrix analysis, in the Korean digital healthcare field, technology development using wearable devices or toilet bowls for identification, diagnosis, and treatment of users and patient pattern analysis using big data are areas where the concentration of $R \& D$ or patent applications is relatively low. Diagnosis through game participant behavior pattern analysis, monitoring using hybrid sensors, and user identification methods can also be classified into areas to be further studied. In addition, various bio-signals have been monitored and used for diagnosis, but it may be necessary to consider using new bio-signals that have not been used so far.

\section{Conclusion}

The Korean healthcare system is shifting from treatment centered on prevention and health management. However, in Korea, due to the adoption of a fee-for-

Table 6. OS Matrix of Korean patents related to healthcare.

\begin{tabular}{ccccccc}
\hline & monitoring & user identification & diagnosis & treatment & convergence & total \\
\hline IoT & 118 & 9 & 12 & 9 & 125 & 273 \\
Mobile Phone & 150 & 20 & 20 & 8 & 22 & 220 \\
Network & 81 & 19 & 28 & 15 & 44 & 187 \\
Health information & 61 & 5 & 12 & 7 & 14 & 99 \\
Big data & 28 & 13 & 14 & 3 & 20 & 78 \\
Wearable device & 51 & 7 & 3 & 3 & 6 & 70 \\
Total & 489 & 73 & 89 & 45 & 231 & 927 \\
\hline
\end{tabular}


service system, insurance premiums are paid according to the number of patients treated by doctors, and there is a problem that insurance premiums are not paid for prevention and health care activities. Furthermore, for healthcare big data, the creation or use of medical big data has been severely restricted due to various regulatory policies such as personal information regulation, public information regulation, specific cloud use regulation, and structural problems for medical services. These problems should be solved one by one.

The total number of patents to be analyzed in this study was 3786, of which 1337 patent applications were filed in Korea, the highest (35.3\%), followed by 935 (24.7\%) in the United States, 746 (19.7\%) in the Japan, 715 cases (18.9\%) in China, and 53 (1.4\%) in Europe. Looking at patents by detailed technology, patents for biometric measurement technology (A1) were searched; the highest was 1243 (32.8\%); secondly, customized teleservice technology (C3) was 688 (18.2\%); followed by Mobile healthcare service platform technology (B3) 460 (12.2\%).

In addition, if the top 10 patent applicants related to healthcare patents are observed, Korean companies account for half; Japan has three companies; and China and US companies each have one company.

As a result of analyzing the patent indices, the field with high patent concentration in Korean healthcare-related technology field is health information building, sharing and utilization technology (B1), and the lowest field is remote monitoring technology $(\mathrm{C} 1)$.

The marketability through patents of Korea is weaker than that of Japan, the United States, and Europe, and the citation index indicating the original patent shows that they do not have many important or original patents. In particular, the field of health information big data analysis technology (B2) is the lowest at 0.59 , indicating that investment in this field is necessary.

In the case of the influence index, which indicates the qualitative level of technologies of Korea, it is lower than that of Japan, but slightly higher than that of the United States and China, so it is considered that Korean patented technology is generally high except for the remote monitoring technology field (C1).

As a result of the OS Matrix analysis, in the Korean digital healthcare field, technology development using wearable devices or toilet bowls for identification, diagnosis, and treatment of users, and patient pattern analysis using big data are areas with relatively low concentration of $\mathrm{R} \& \mathrm{D}$ or patent applications. It appears, and it seems that technology development and patent application for these fields will be needed in the future.

In our study, five indices such as AI, PFS, CPP, PII, and TS were analyzed. In future studies, the more patent indices, for example, TCT, CRn, HHI, etc., should be used for a precise analysis. And research on a tool that presents more accurate future business items through OS matrix analysis is needed.

\section{Acknowledgements}

This study was supported by Gangneung-Wonju National University (GWNU 2018-08). 


\section{Conflicts of Interest}

The authors declare no conflicts of interest regarding the publication of this paper.

\section{References}

Health Insurance Review and Assessment Service. http://www.hira.or.kr

Jung, D.-H., Kwon, O.-Y., \& Koo, Y.-D. (2018). Analysis of Causes and Characteristics of Death in Gangwon-do Using Medical Big Data. Journal of the Korea Contents Association, 18, 149-155.

Jung, K.-H., Kim, G.-H., Park, J.-W., Lee, H.-S., Kim, H.-J., Choi, H.-Y., \& Kim, J.-H. (2015). Prediction and Cause Analysis of Patients' Rehospitalization through Medical Data Mashup and Big Data Technique. Enture Journal of Information Technology, 14, 133-149.

Jung, M.-J., \& Lee, M.-H. (2017). Medical Big Data Activation Policy for the Development of the Healthcare Industry. Integrated Journal of the Korean Management Association, 2017, 1658-1677.

Kim, D.-S., Cho, S.-H., Lee, J.-S., Kim, M.-S., \& Kim, N.-H. (2018). A Study on the Competitiveness Analysis of Korea's Digital Healthcare Field through Patent Analysis. Journal of the Korea Digital Policy Association, 16, 229-237.

Kim, H.-J., Jung, S.-H., Lee, G.-H., \& Cho, W.-S. (2017). Health Care Big Data Connection and Standardization Process to Protect Personal Information. Korean Contents Association 2017 Spring Conference, Daejeon, 5 December 2017, 31-32.

Kim, J. (2019). Big Data Utilization and Personal Information Protection in the Health and Medical Field. Law \& Technology, 15, 49-67.

Kim, J.-H. (2017). MapReduce-Based Health Care Service Using Big Data. Proceedings of the 2017 Korea Software Conference, 1732-1733.

Kim, Y., Kim, S., Cho, M., \& Kim, W. (2014). Building a Big Data Processing Environment for Learning Systems. JKIECS, 9, 791-798.

https://doi.org/10.13067/JKIECS.2014.9.7.791

Korea Institute for Health and Social Affairs. http://www.kihasa.re.kr

Korea Statistics (2015). Current Status and Prospect of Population in the World and Korea (pp. 1-37).

Laney, D. (2001). 3D Data Management: Controlling Data Volume, Velocity, and Variety. Application Delivery Strategies, Gartner.

Lim, M., Choi, J., Jung, H., Han, Y., \& Park, W. (2017). Prediction of Ensemble of Health Numerical Values Using Deep Learning Techniques. Joint Conference of Korean Digital Contents Society and Korea Information Technology Society, Busan, 21 December 2017, 197-198.

National Cancer Center. http://www.ncc.re.kr

National Health Insurance Service. http://www.nhis.or.kr

Nkenyereye, L., \& Jang, J. W. (2015). Development of Big Data Solution for Smart Car Service Using Hadoop. Journal of the Korea Information and Communication Society, 19, 607-612. https://doi.org/10.6109/jkiice.2015.19.3.607

Shim, M.-R., \& Shim, H.-J. (2019). Legal Issues in the Use of Medical Information for the Development of the Bio-Health Industry. Journal of Industrial Property, 58, 1-47.

Shin, S.-Y. (2017). Current Status of Domestic Healthcare Big Data and New Research Directions. Journal of Information Science, 35, 16-19.

The Korea Disease Control and Prevention Agency. http://www.kdca.go.kr/ 